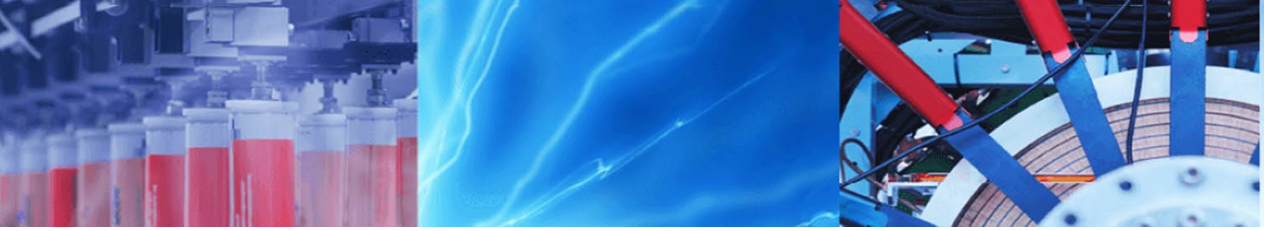

Research Article

\title{
Reliability research of reference temperature estimation of RPV materials using Charpy impact energy
}

\author{
Mengle Yin ${ }^{1}$ Jianhua Pan ${ }^{1,2}$ (1)
}

Received: 13 October 2019 / Accepted: 23 November 2019 / Published online: 6 December 2019

(c) Springer Nature Switzerland AG 2019

\begin{abstract}
The standard fracture toughness test is usually limited in practical engineering projects due to the expensive and sophisticated experimental procedure or limited specimens. The traditional method to evaluate the structural integrity is to estimate the ductile-brittle transition region by Charpy tests. However, this fuzzy estimation is sometimes too conservative and cannot obtain quantitative fracture toughness data. In recent years, many achievements have been made in the study of the relations between Charpy impact test and fracture toughness test. Although acceptable, the errors caused by these formulas are rarely compared in detail formulas with stable deviation will be more suitable for research or engineering application. Therefore, this paper compares the data of four different ferritic steels to test the error stability of each formula, so as to provide reference for experimental research and engineering facilities. The reliability of each correlation is checked from the view of accuracy or appropriate conservatism. In this research, We found that the $T_{28}-T_{0}$ correlations, the empirical formula proposed by Rolfe, Novak and Barsom (abbreviated as RNB) and the Mean-4 Procedure obtained by IGC-parameter give the reference temperatures more accurately or appropriately conservatively. These formulas should be preferred in practical application.
\end{abstract}

Keywords Charpy impact test $\cdot$ Empirical formula $\cdot$ Reference temperature $\cdot$ RPV materials $\cdot$ IGC-parameter

\section{Introduction}

Ferritic steels have obvious ductile-brittle transition behavior. The fracture mode is brittle fracture when the temperature is lower than a certain range and is ductile fracture when the temperature is higher than that range. Within this range, the fracture mode gradually transits from brittle fracture to ductile fracture with the increase of temperature. This temperature range is named as the ductile-brittle transition region. Ferritic steels hardly undergo plastic deformation before the brittle fracture occurs. Therefore, brittle fracture is instantaneous and difficult to prevent. Moreover, the unstable propagation of crack hardly requires external force. Consequence is expected to be very serious once brittle fracture occurs. To avoid brittle fracture of ferritic steels, the lower working temperature limit must be higher than the transition region. Under long-term irradiation, the transition region will move toward higher temperature due to the neutron irradiation embrittlement [1]. To ensure the structural integrity and service life of reactor pressure vessels, precise fracture-safe analysis is very important and necessary.

Master Curve method is a widely used technique to research and analyze fracture toughness. This method characterizes the distribution of fracture toughness in transition region as a curve determined by the reference temperature. The lower bound of fracture toughness is given by measuring and calculating the reference

\footnotetext{
$\triangle$ Jianhua Pan, panjianhua_123@163.com | ${ }^{1}$ Anhui Province Key Lab of Aerospace Structural Parts Forming Technology and Equipment, Institute of Industry and Equipment Technology, Hefei University of Technology, Hefei 230009, China. ${ }^{2}$ National Engineering and Technology Research, Center on Pressure Vessel and Piping Safety, Hefei General Machinery Research Institute, Hefei 230031, China.
} 
temperature. ASTM E1921 provides methods to determine the reference temperature, including a single-temperature method and a multi-temperature method [2]. However, fracture toughness test is complex and expensive because of the requirements of stringent procedures, specialized testers and thick specimens with sharp fatigue pre-crack [3]. In addition, limited specimens provided by nuclear facilities may not meet the requirement of fracture toughness test. One of the ways to solve this problem is to use small size samples. But the size effect makes the measured fracture toughness deviate from the normal value [4]. Therefore, the methods by transforming a non-SSY value of $K_{J C}$ to an equivalent SSY value have been researched in recent years [5]. But without appropriate official standards, these studies cannot be applied to practical projects yet.

The structural integrity evaluation of important facilities is often based on the experimental results of small samples, such as fracture toughness test, Charpy impact test, small punch test, etc. [6,7]. Charpy impact test is a traditional test used to determine the notch sensitivity of metal materials. Compared with the standard fracture toughness test prescribed by ASTM, Charpy impact test has lower requirements for sample preparation and equipment. The sensitivity to material quality, defects and structure is also not available in static load test. In addition, Charpy impact test requires fewer specimens. Fractured Charpy impact specimens can be reconstituted and reused $[8,9]$. This technology makes it possible to obtain more test data with a small number of specimens. However, the test data obtained from Charpy impact test (mainly impact energy) cannot directly represent the fracture toughness or reference temperature. Sometimes, determining the temperature shift by the impact test is too conservative. The differences of specimen size and strain rate effect [10] between Charpy impact test and fracture toughness test also complicate the relationship between impact energy and fracture toughness.

In the past few decades, Charpy impact test was the main way to evaluate RPV materials and a large number of impact test data have been accumulated. Many correlations between impact energy and fracture toughness have been established, such as formulas for estimating the reference temperature directly with $28 \mathrm{~J}$ or $41 \mathrm{~J}$ impact energy proposed by Sattari-Far and Wallin [11], or the empirical formula for estimating fracture toughness with the impact energy established by Rolfe, Novak and Barsom, etc. [12, 13]. Nowadays, with the development of computer technology, finite element analysis (FEA) has been applied increasingly to crack simulation and the prediction of fracture toughness [14-16]. The finite element method also makes it possible to obtain fracture toughness data by tensile test.[17] It greatly simplifies the acquisition of fracture toughness data. But the finite element analysis cannot be applied to practical engineering project yet. Charpy impact test is still the main way to determine the ductile brittle transition temperature (DBTT). These Charpy-fracture toughness formulas have been established and tested for many years. They all have inevitable errors. Although recognized, there is not much work to study and compare these errors. If these formulas need to be applied to practical projects, it will be very helpful to know the degree of their errors. An empirical formula with stable error is of great significance to both practical engineering and academic research.

In this paper, several different formulas are applied to four RPV materials. The calculated results are compared with the results of the standard fracture toughness test and their deviations are compared. The comparison results will be discussed from the view of magnitude and stability of the deviation, as well as the complexity of the calculation process. The purpose of this paper is to find out the most suitable fracture toughness estimation formula for ferritic steels, and to provide reference for practical engineering and academic research.

\section{Relations between reference temperature and Charpy impact energy}

\subsection{Relations between reference temperature and $T_{28}$ or $T_{41} \mathrm{~J}$}

$28 \mathrm{~J}$ and $41 \mathrm{~J}$ Charpy energy temperature are often used to define DBTT (ductile brittle transition temperature). They can also be used to estimate the reference temperature. Some simple formulas for estimating reference temperature are shown in Table 1.

Wallin proposed the relations between reference temperature and $T_{28}$ or $T_{41}$ (i.e. Equations (2) and (4), and the corresponding conservative formulas (i.e. Eqs. (2a) and (4a)) in [11]. The relation between $T_{28}$ and reference temperature is also written as Eq. (1) in [18]. Equations (1) is close to Eq. (2), and only Eq. (1) with smaller standard

Table 1 Relations between reference temperature and $T_{28}$ or $T_{41 \mathrm{~J}}$

\begin{tabular}{ll}
\hline Formulas & Eq. nos. \\
\hline$T_{0}=T_{28 \jmath}-18\left(\sigma=15^{\circ} \mathrm{C}\right)$ & (1) \\
$T_{0}=T_{28 \jmath}-19\left(\sigma=22^{\circ} \mathrm{C}\right)$ & (2) \\
$T_{0-1 \sigma}=T_{28 \jmath}+3$ & (2a) \\
$T_{0}=1.09 \cdot T_{28 \jmath}-11.2\left(\sigma=18^{\circ} \mathrm{C}\right)$ & (3) \\
$T_{0-1 \sigma}=1.18 \cdot T_{28 J}-12.52$ & (3a) \\
$T_{0}=T_{41 \jmath}-26\left(\sigma=25^{\circ} \mathrm{C}\right)$ & (4) \\
$T_{0-1 \sigma}=T_{41 J}-1$ & (4a) \\
\hline
\end{tabular}

All the temperatures in this table are in ${ }^{\circ} \mathrm{C}$ 
deviation is usually considered. Equations (3-3a) are other formulas between $T_{28}$ and reference temperature provided by Sreenivasan [19]. These formulas will be used in this paper as well. In [19], the non-linear correlations between reference temperature and $T_{28}$ or $T_{41}$ are also studied. The corresponding non-linear formulas are developed as shown in Eqs. (11-12) in Table 3.

\subsection{One-step or two-step empirical formulas}

Similar to the ductile-brittle transition curve, the Charpy transition curve can also be divided into three regions: upper shelf, transition region and lower shelf. Empirical formulas cannot describe all three regions at the same time because of the complexity of the Charpy transition curve. Empirical formulas are only applicable to one or two of the three regions. In this paper, only empirical formulas applicable to transition region are discussed.

Some commonly used one-step or two-step empirical methods applicable to transition region are shown in Table 2. In Table 2, $K_{I C}$ or $K_{D}$ is the Static or dynamic fracture toughness in $\mathrm{MPa} \sqrt{\mathrm{m}}$ and $C_{V}$ is the Charpy impact energy in J. $\sigma_{y s-R T}$ is the yield strength at room temperature in $\mathrm{MPa}$ and $\mathrm{E}$ is Young modulus in $\mathrm{GPa}$. The corresponding fracture toughness transition curves can be obtained by applying these empirical formulas (i.e. Eqs. (5-9)) once the Charpy transition curves are available. Usually, the temperature corresponding to a fracture toughness of $100 \mathrm{MPa} \sqrt{\mathrm{m}}$ will be used as an estimation of the reference temperature. To distinguish the estimated results conveniently, the reference temperatures calculated by this method are recorded as $T_{K 100-X} . X$ is the initials of the sources of the empirical formulas. Therefore, the four empirical formulas are abbreviated as RNB [12, 13], SC [20], RLB [21] and BR [12]. The estimated reference temperatures of Eqs. (5-9) obtained by this method are $T_{K 100-R N B}, T_{K 100-S C}, T_{K 100-R L B}, T_{K 100-B R K D}$ (calculated by Eq. (8) alone) and $T_{K 100-B R S}$ (calculated by Eqs. (8-9)) respectively.

Strictly speaking, the fracture toughness represented by $T_{K 100-X}$ represents the fracture toughness of specimens
Table 3 Non-linear fitting formulas for reference temperature

\begin{tabular}{ll}
\hline Formulas & $\begin{array}{l}\text { Eq. } \\
\text { nos. }\end{array}$ \\
\hline$T_{Q-28}=-222.55+217.64 \cdot \exp \left(0.0062 \cdot T_{28 J}\right)$ & (11) \\
$T_{Q-41}=-275.28+252.19 \cdot \exp \left(0.00463 \cdot T_{41 J}\right)$ & (12) \\
$T_{Q-R N B}=\left(-14.25+T_{K 100-R N B}\right) /\left(0.8166-0.0019 \cdot T_{K 100-R N B}\right)$ \\
$T_{Q-S C}=\left(-25.67+T_{K 100-S C}\right) /\left(0.8713-0.0019 \cdot T_{K 100-S C}\right)$ \\
$T_{Q-R L B}=\left(-29.3+T_{K 100-R L B}\right) /\left(0.8987-0.0018 \cdot T_{K 100-R L B}\right)$ \\
$T_{Q-I G C 1}=\left(-55.33+P_{I G C 1}\right) /\left(2.42-0.0028 \cdot P_{I G C 1}\right)$ \\
$T_{Q-I G C 2}=\left(-21.578+P_{I G C 2}\right) /\left(0.8498-0.0033 \cdot P_{I G C 2}\right)$
\end{tabular}

All the temperatures in this table are in ${ }^{\circ} \mathrm{C}$

with thickness of $10 \mathrm{~mm}$. Because of the lower thickness, the calculated fracture toughness is higher than the actual one and the corresponding reference temperature is lower as well. The fracture toughness values of different thickness can be normalized to those of 1T thickness by Eq. (10). Thickness effect is not negligible. Omitting thickness normalization simplifies the calculation process. But it is still necessary to discuss the influence. The 1T-equivalent fracture toughness $\left(K_{J C}\right)$ transition curve can be calculated by using Eq. (10). For convenience of distinction, the temperature corresponding to $1 \mathrm{~T}$-equivalent fracture toughness of $100 \mathrm{MPa} \sqrt{\mathrm{m}}$ is recorded as $T_{K 100-X(17)}$. The subscript (1T) indicates that the equivalent thickness is $1 \mathrm{~T}$.

$K_{J C(1 T)}=20+\left(K_{J C}-20\right) \cdot\left(\frac{B}{B_{0}}\right)^{1 / 4}$

$B$ is $10 \mathrm{~mm}$ and $B_{0}$ is $25.4 \mathrm{~mm}$ for standard Charpy impact specimens.

Omitting thickness normalization may enlarge the error. The non-linear fitting formulas between $T_{K 100-x}$ and $T_{0}$ proposed in [19] may be helpful for error reduction. As shown in Eqs. (13-15) in Table 3, the reference temperatures calculated by using the fitting formulas are termed
Table 2 One-step or two-step empirical formulas

\begin{tabular}{lllll}
\hline Sources & Formulas & \multicolumn{2}{l}{ Suitable Range } & Eq. nos. \\
\cline { 3 - 5 } & & $C_{V}(\mathrm{~J})$ & $\sigma_{y s-R T}(\mathrm{MPa})$ \\
\hline Rolfe, Novak and Barsom & $K_{I C}=\left(E \cdot 1000 \cdot(2.28) \cdot 10^{-4} \cdot C_{V}^{1.5}\right)^{0.5}$ & $4-82$ & $270-1700$ & $(5)$ \\
Sailors and Corten & $K_{I C}=14.63 \cdot C_{V}^{0.5}$ & $7-68$ & $410-480$ & $(6)$ \\
Robert's lower-bound correlation & $K_{I C}=8.47 \cdot C_{V}^{0.63}$ & - & - & $(7)$ \\
Barsom and Rolfe & $K_{D}=\left(0.64 \cdot E \cdot C_{V}\right)^{0.5}$ & $2.7-61$ & $250-345$ & $(8)$ \\
& $T_{\text {shifted }}=T_{C V N}-\left(119-0.12 \cdot \sigma_{y s-R T}\right)$ & & & (9) \\
\hline
\end{tabular}

Where $K_{I C}$ or $K_{D}$ is the Static or dynamic fracture toughness in $\mathrm{MPa} \sqrt{\mathrm{m}}$ and $C_{V}$ is the Charpy impact energy in J. $\sigma_{y S-R T}$ is the yield strength at room temperature in $\mathrm{MPa}$ and $\mathrm{E}$ is Young modulus in $\mathrm{GPa}$ 
as $T_{Q-X}$ and subscript $X$ represents the initials of empirical formulas as well.

\subsection{Relations between reference temperature and IGC-parameter}

Based on Miyata and Tagawa's research [22] about the relations between micro-cleavage fracture stress $\left(\sigma_{f}\right.$ a given steel constant), yield stress and fracture toughness, Sreenivasan defined two new parameters, $P_{I G C 1}$ and $P_{\text {IGC2 }}$ [19], as follows:

$P_{I G C 1}=\left(\frac{\sigma_{f}}{\sigma_{y S-R T}}\right) \cdot T_{41 J}$

$P_{I G C 2}=\left(\frac{\sigma_{y s-T 41}}{\sigma_{y s-R T}}\right) \cdot T_{41 J}$

where $T_{41}$ is the $41 \mathrm{~J}$ Charpy energy temperature and $\sigma_{y s-T 41}$ is the yield strength at $T_{41}$. $\sigma_{y s-R T}$ is the yield strength at room temperature. According to Miyata and Tagawa's research, $\sigma_{f}$ can be estimated using $K_{l C}-\sigma_{y s}$ or $K_{l d}-\sigma_{y d}$ data at very low temperature (about $-150^{\circ} \mathrm{C}$ ) by solving the following Eq. (20) [19].

$\left.0=\left(\frac{K_{I C-T 1}}{K_{I C-T 2}}\right)-\left(\frac{\sigma_{y s-T 1}}{\sigma_{y s-T 2}}\right) \frac{\sqrt{\exp \left(\frac{\sigma_{f}}{\sigma_{y s-T 1}}-1\right)-1}}{\sqrt{\exp \left(\frac{\sigma_{f}}{\sigma_{y s-T 2}}-1\right)-1}}\right)$

where $K_{l C}-\sigma_{y s}$ values at two temperatures are used. If there is sufficient data, multiple groups of $\sigma_{f}$ can be obtained with the temperature interval of $10-15^{\circ} \mathrm{C}$. The mean value is taken if $\sigma_{f}$ values agree within $10 \%$ [19]. $P_{I G C 1}$ and $P_{I G C 2}$ can be used to estimate the reference temperatures with Eq. (16-17) [19] and the calculated results are expressed as $T_{Q-I G C 1}$ and $T_{Q-I G C 2}$ respectively.

\section{Materials and experimental data}

\subsection{Stress-strain relations of the materials}

Four commonly used RPV materials are investigated in this paper. The grades are China A508-3, USA A533B, Euro $20 \mathrm{MnMoNi} 55$ and $16 \mathrm{MnDR}$ and the chemical compositions of these materials $[23,24]$ are listed in Tables 4 and 5 .

Tensile test is widely used to measure the mechanical properties of materials, such as Young modulus, yield strength, etc. These data are also necessary to evaluate the structural integrity. To obtain the mechanical properties of $16 \mathrm{MnDR}$, the tensile test was carried out in this paper. Specimens were prepared according to GB/T 2975-1998 [25]. The test was carried out on CMT5105 computer controlled electronic universal testing machine. Before the test start, the specimen was kept at the target temperature for at least 100 min to ensure the uniformity of the temperature. The test temperature ranged from $-100^{\circ} \mathrm{C}$ to room temperature and the loading rate was $2 \mathrm{~mm} / \mathrm{min}$. The specimen size and experimental data used in this paper are shown in Figs. 1 and 2.

The yield strength data of $16 \mathrm{MnDR}$ and the rest three materials [26] are fitted and shown in Eqs. (21-24) respectively:

A508-3: $\sigma_{y s}(\mathrm{MPa})=0.0013 \cdot T^{2}-0.6171 \cdot T+462.58$

A533B $: \sigma_{y s}(\mathrm{MPa})=0.0074 \cdot T^{2}-0.2386 \cdot T+493.1278$

20MnMoNi55: $\sigma_{y s}(\mathrm{MPa})=0.0112 \cdot T^{2}-0.0431 \cdot T+494.01$

$16 \mathrm{MnDR}: \sigma_{y s}(\mathrm{MPa})=380.41-0.6633 \cdot T$

where $\sigma_{y s}$ is yield strength in $\mathrm{MPa}$ and $\mathrm{T}$ is in ${ }^{\circ} \mathrm{C}$.

Table 4 Chemical composition of three materials/ \%

\begin{tabular}{llllllllllll}
\hline Material Brand & \multicolumn{2}{l}{ Chemical Composition } & \multicolumn{1}{l}{} & & & & \\
& \cline { 2 - 11 } & $\mathrm{C}$ & $\mathrm{Si}$ & $\mathrm{Mn}$ & $\mathrm{Ni}$ & $\mathrm{Cr}$ & $\mathrm{Mo}$ & $\mathrm{P}$ & $\mathrm{S}$ & $\mathrm{Cu}$ & $\mathrm{V}$ \\
\hline A533B & $\leq 0.25$ & $0.15-0.40$ & $1.15-1.50$ & $0.40-0.70$ & - & $0.45-0.60$ & $\leq 0.035$ & $\leq 0.035$ & $\leq 0.10$ & $\leq 0.05$ \\
20MnMoNi55 & $0.17-0.23$ & $0.15-0.30$ & $1.20-1.50$ & $0.50-1.00$ & $\leq 0.20$ & $0.40-0.60$ & $\leq 0.012$ & $\leq 0.015$ & $\leq 0.10$ & $\leq 0.02$ \\
China A508-3 & $0.16-0.20$ & $0.10-0.30$ & $1.20-1.55$ & $0.50-0.80$ & $\leq 0.20$ & $0.45-0.55$ & $\leq 0.008$ & $\leq 0.008$ & $\leq 0.08$ & $\leq 0.01$ \\
\hline
\end{tabular}

Table 5 Chemical composition of $16 \mathrm{MnDR} / \%$

\begin{tabular}{lllllll}
\hline Material Brand & \multicolumn{5}{l}{ Chemical Composition } \\
\cline { 2 - 7 } & $\mathrm{C}$ & $\mathrm{Si}$ & $\mathrm{Mn}$ & $\mathrm{Al}_{\mathrm{t}}$ & $\mathrm{P}$ & $\mathrm{S}$ \\
\hline $16 \mathrm{MnDR}$ & $\leq 0.20$ & $0.15-0.50$ & $1.20-1.60$ & $\geq 0.02$ & $\leq 0.025$ & $\leq 0.012$ \\
\hline
\end{tabular}


Fig. 1 16MnDR: Sample size of tensile test

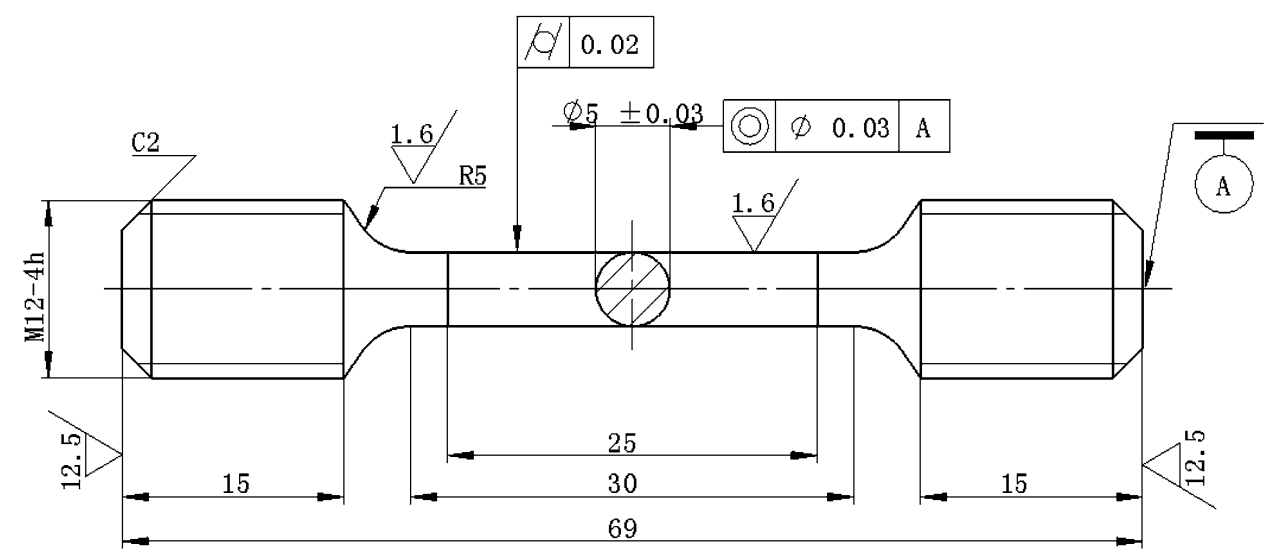

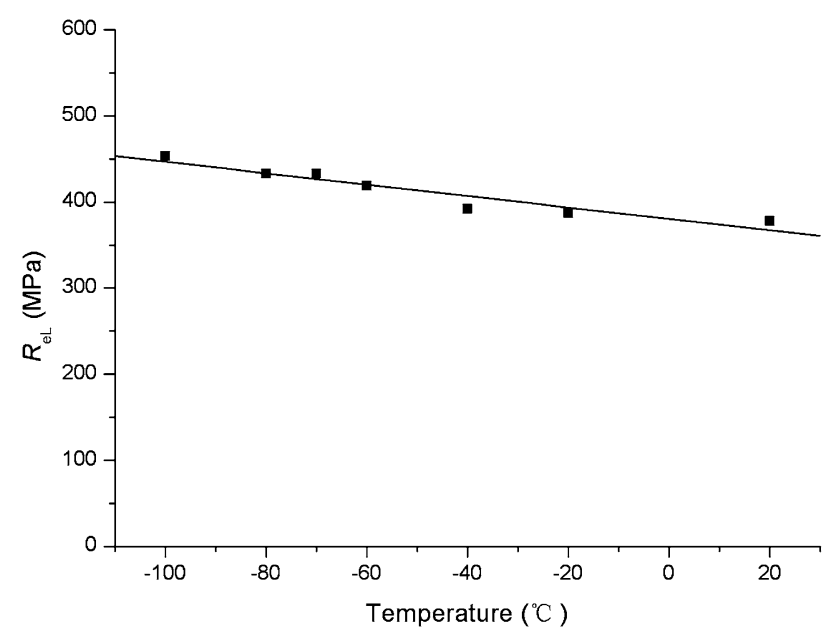

Fig. 2 16MnDR: Fitting curve of yield strength

\subsection{Charpy impact data of the materials}

Charpy impact test is a Commonly used test to check the dynamic mechanical properties of materials. It measures the absorbed energy when a pendulum breaks a sample of a certain shape. Although not very precisely, the Charpy impact energy can be used to determine the transition region conveniently as the basis for evaluating the service life and security of RPV. In lower shelf and upper shelf, the fracture modes of ferritic steels are mainly brittle fracture and ductile fracture respectively with relatively stable values of Charpy impact energy. In transition region, the fracture mode gradually changes from brittle fracture to ductile fracture with the increase of temperature. The impact energy data are also more dispersed. According to Oldfield's research [27], the Charpy impact energy data can be fitted into a tangent hyperbolic function.

The Charpy impact test of $16 \mathrm{MnDR}$ was performed under the guidance of the standard GB/T 229-2007 [28]. Standard impact specimens of $10 \mathrm{~mm} * 10 \mathrm{~mm}$ size were prepare

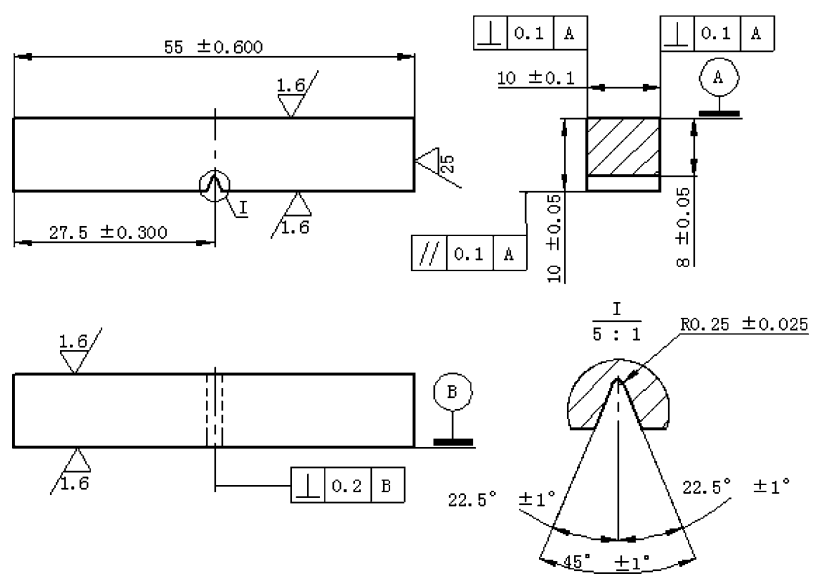

Fig. 3 16MnDR: Sample size of Charpy impact test

according to GB/T 2975-1998 [25]. The detailed geometric size of the specimen is shown in Fig. 3. V-shaped notch with depth of $2 \mathrm{~mm}$ was processed in the middle of the specimen. The impact test was conducted in the JBD-300A low temperature impact tester. The radius of the pendulum blade was $2 \mathrm{~mm}$. In the experiment, the pendulum had about $5 \mathrm{~m} / \mathrm{s}$ loading velocity and $300 \mathrm{~J} \mathrm{impact} \mathrm{energy.} \mathrm{The} \mathrm{experi-}$ mental temperature ranged from -100 to $-20^{\circ} \mathrm{C}$ and the test temperatures were maintained with liquid nitrogen and anhydrous ethanol solution. As shown in Fig. 4, the impact data are fitted into a tangent hyperbolic curve.

The Charpy transition curves for $16 \mathrm{MnDR}$ and the rest three materials [26] are shown in Eqs. (25-28) respectively.

$$
\begin{aligned}
& \text { A508-3: } E_{C V N}=130.89+123.22 \cdot \tanh \left(\frac{T+17.00}{28.10}\right) \\
& \text { A533B }: E_{C V N}=113.68+90.36 \cdot \tanh \left(\frac{T+4.81}{21.88}\right)
\end{aligned}
$$




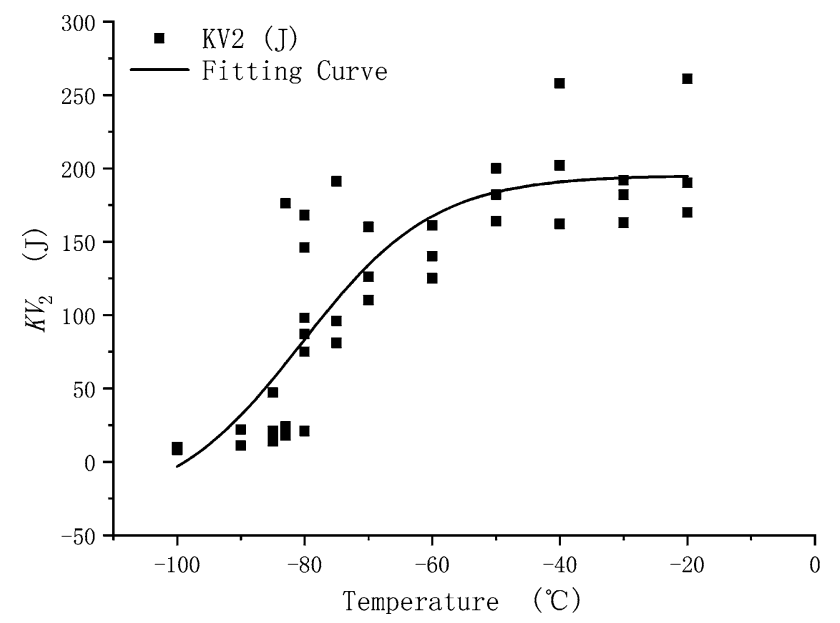

Fig. 4 16MnDR: The Charpy transition curve

$20 \mathrm{MnMoNi55}: E_{C V N}=117.07+88.00 \cdot \tanh \left(\frac{T+48.40}{29.36}\right)$

$16 \mathrm{MnDR}: E_{C V N}=81.12+114.05 \cdot \tanh \left(\frac{T+80.4}{20.65}\right)$

where $E_{C V N}$ is the Charpy impact energy in $\mathrm{J}$ and $T$ is in ${ }^{\circ} \mathrm{C}$. The Charpy transition curves will be used to estimate the reference temperature in the following research.

\subsection{Young modulus of the materials}

In the process of calculation, the influence of temperature on Young modulus should be taken into account. The relationship between Young modulus and temperature commonly used in ferritic steels is provided in [29], as shown in Eq. 29.

$E=207.2-0.0571 \cdot T\left({ }^{\circ} \mathrm{C}, \mathrm{GPa}\right)$

\section{Results and discussion}

\subsection{Estimations of reference temperature by directly use of $\mathrm{T}_{28 \mathrm{~J}}$ or $\mathrm{T}_{41 \mathrm{~J}}$}

In this section, the relations between $T_{0}$ and $T_{28}$ or $T_{41} \mathrm{~J}$ shown in Eqs. (1-4a) are mainly researched and compared. $T_{28}$ and $T_{41}$ are obtained directly by the Charpy transition curves. The estimated results are recorded as $T_{0(1)}, T_{0(2 a)}$, $T_{0(3)}, T_{0(3 a)}, T_{0(4)}$ and $T_{0(4 a)}$ with subscript denoting the serial numbers of the corresponding formulas respectively. The reference temperatures calculated by Eqs. (11-12) are recorded as $T_{\mathrm{Q}-28}$ and $T_{\mathrm{Q}-41}$ respectively. In this paper, the deviations (expressed in $\Delta T_{0}$ and equal to estimated

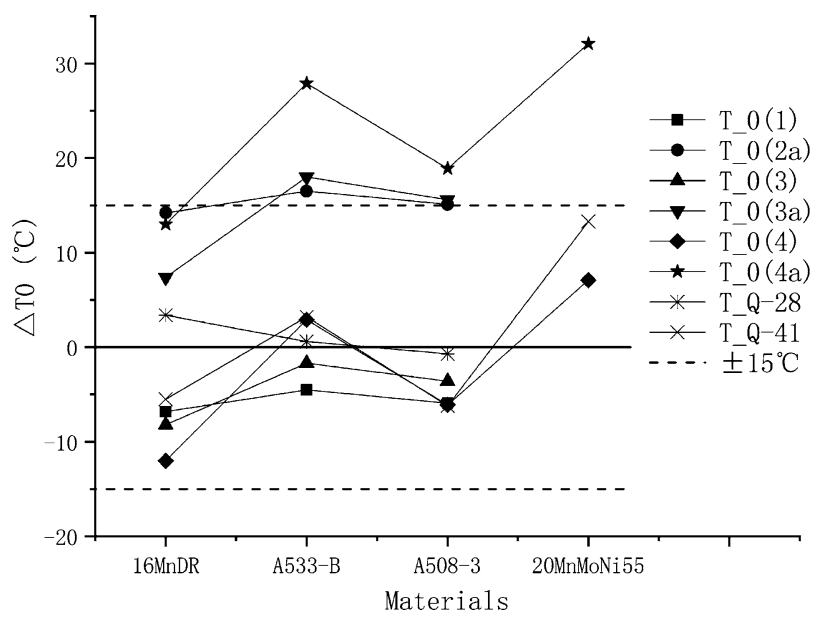

Fig. 5 Deviations of reference temperatures estimated by $T_{28 ر}$ or $T_{41 \mathrm{~J}}$

temperature minus tested temperature) between the estimated temperatures and tested temperatures are compared. All the deviations are plotted in Fig. 5 with dashed lines representing the deviation of $\pm 15^{\circ} \mathrm{C}$.

According to the relevant literature [24], the tested $T_{0}$ of $16 \mathrm{MnDR}$ obtained by the fracture toughness test is $-102^{\circ} \mathrm{C}$. Reference temperatures of China A508-3 and USA $\mathrm{A} 533 \mathrm{~B}$ are considered as $-63^{\circ} \mathrm{C}$ and $-58^{\circ} \mathrm{C}$ [26] respectively. Reference temperature of Euro $20 \mathrm{MnMoNi55}$ is between -120 and $-130{ }^{\circ} \mathrm{C}$ [26] and the median value $-125^{\circ} \mathrm{C}$ is used for calculation and comparison in this paper.

According to the data in Fig. 5, most of the deviations fluctuate within certain ranges. Those stable trends reveals that there is a relatively stable relationship between $T_{0}$ and $T_{28}$ or $T_{41}$. In Fig. 5, the results calculated by $T_{28 \mathrm{~J}}\left(T_{0(1)}\right.$ to $T_{0(3 a)}$ and $T_{Q-28}$ ) obviously have smaller fluctuation ranges than that calculated by $T_{41}$. This trend indicates that $T_{28} \mathrm{~J}$ can reflect the reference temperatures more accurately than $T_{41}$. However, some materials can hardly obtain $28 \mathrm{~J}$ impact energy because mostly the $T_{28}$ falls on the lower shelf. The formulas of $T_{28}$, cannot be applied to all materials.

By comparing the estimated results of each formula, $T_{Q-28}$ and $T_{0(2 a)}$ are noticed to have the smallest deviation amplitude. This phenomenon makes them the preferred choices for simple prediction of reference temperature. The former can provide an accurate prediction while the latter is relatively conservative. The data of Charpy impact test have a wide range of dispersion, especially in transition region. The reference temperatures estimated by Charpy impact tests have greater error than that estimated by fracture toughness tests. In addition, the research of fracture toughness by samples is affected by 
some uncertainties [30]. For safety reasons, the estimated reference temperatures selected in practical engineering should tend to be appropriately conservative. Therefore, the latter one is preferred for practical engineering projects. But these two formulas are not suitable for all situations. Neither $T_{Q-28}$ nor $T_{0(2 a)}$ can be obtained for materials that cannot obtain the $T_{28}$ temperature. In that case, although the errors are slightly larger, conservative $T_{0(4 a)}$ and relatively accurate $T_{Q-41}$ can also be used as backup options.

\subsection{Estimations of reference temperature by empirical formulas}

This section is devoted to discussing the estimation trends of several empirical formulas. The estimated results of each empirical formula are plotted in a single graph, as shown in Figs. 7, 8, 9 and 10 respectively. The $T_{K 100-x^{\prime}} T_{K 100-X(1 T)}$ and $T_{Q-X}$ mentioned above can be calculated directly by combining the Charpy transition curves with empirical formulas and the fitting formulas in Table 3.

The multi-temperature formula in Eq. (30) requires several fracture toughness at different temperatures. These fracture toughness data can be obtained by various empirical formulas. The multi-temperature formula is used to calculate the reference temperature in master curve method. At least six pairs of valid fracture toughness data and the corresponding temperatures are required. The Charpy transition curve, empirical formula and the thickness normalization shown as Eq. (10) can be combined into the fracture toughness - temperature relation of specimens with equivalent thickness of $1 \mathrm{~T}$. The data used in multi-temperature method are taken from here and recorded as $K_{I C(1)}$. Data can be selected in the range of $50 \mathrm{MPa} \sqrt{\mathrm{m}} \leq K_{I C(1 T)} \leq 150 \mathrm{MPa} \sqrt{\mathrm{m}}[26]$. The selection

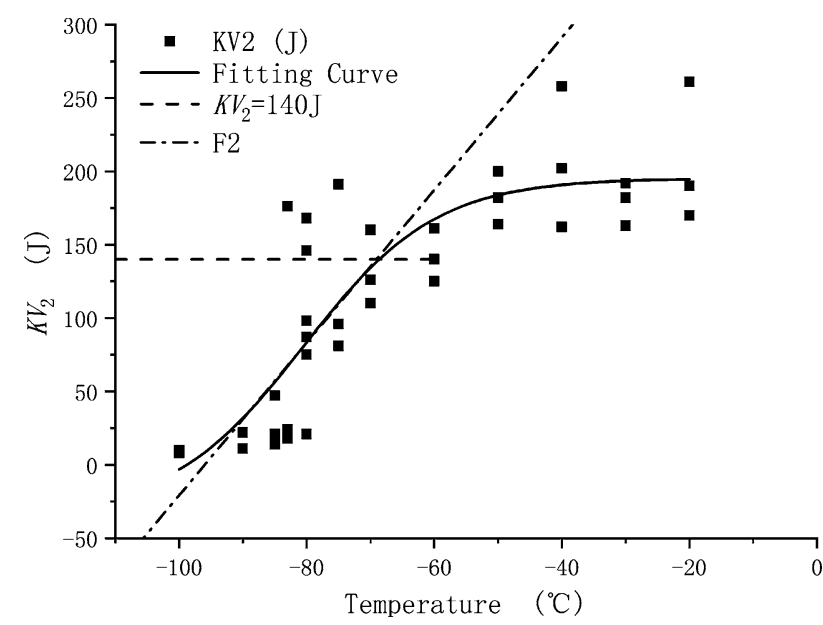

Fig. 6 Transition threshold of 16MnDR

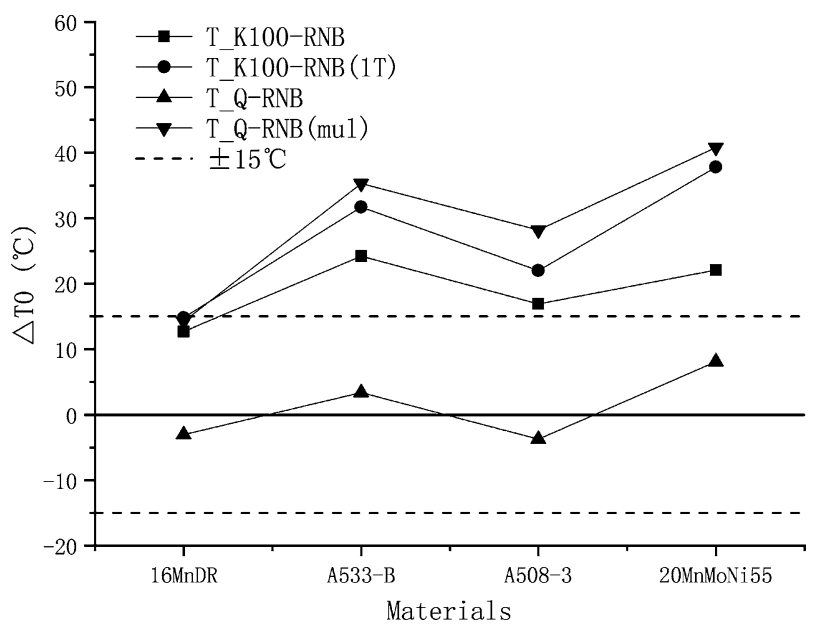

Fig. 7 Deviations of reference temperatures estimated by RNB

of these data must meet the requirements of the multitemperature formula.

The empirical formula applicable to transition region is usually not considered to be applicable to the upper shelf. Therefore, the selected data should not be affected by the impact energy of the upper shelf. The threshold of fracture toughness data can be set according to the Ref. [31]. Taking the $16 \mathrm{MnDR}$ transition curve in Fig. 6 as an example, an eye-fit straight line (the dotted line in Fig. 6) is drawn from the middle of the impact energy curve. The trend of separating the straight line from the impact energy curve starts at the position with about $140 \mathrm{~J}$ impact energy. Thus, the impact energy of the upper shelf must be higher than $140 \mathrm{~J}$. The $140 \mathrm{~J}$ can be used as the threshold of transition region. When selecting fracture toughness data, the corresponding impact energy should not exceed $140 \mathrm{~J}$.

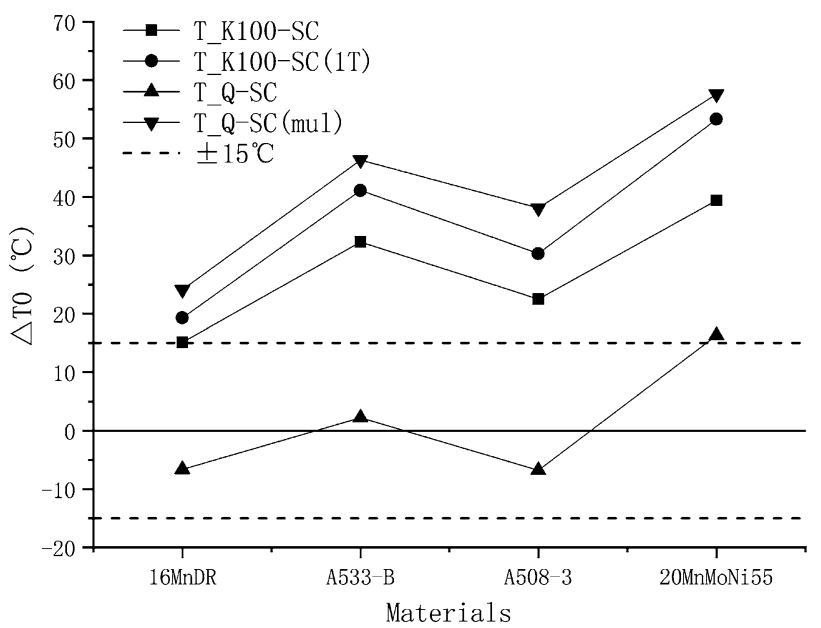

Fig. 8 Deviations of reference temperatures estimated by SC 


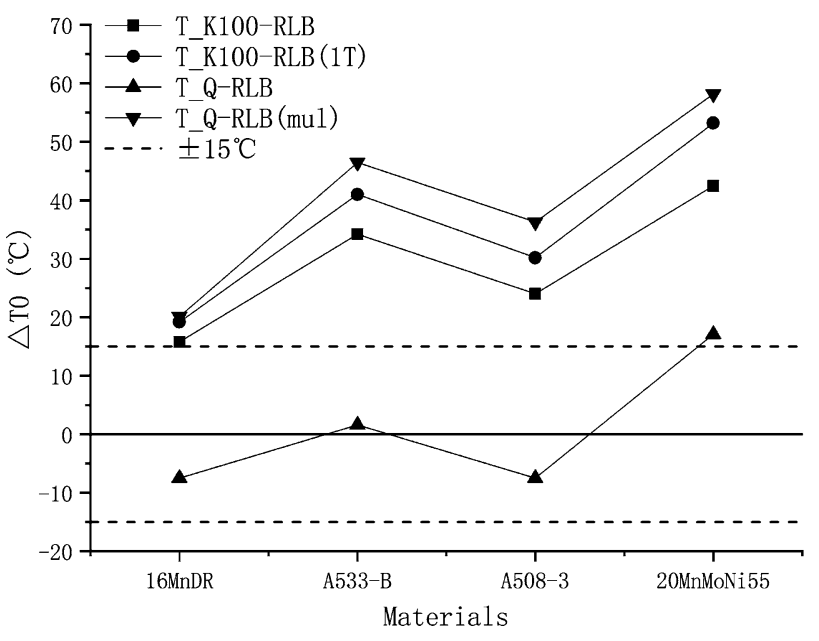

Fig. 9 Deviations of reference temperatures estimated by RLB

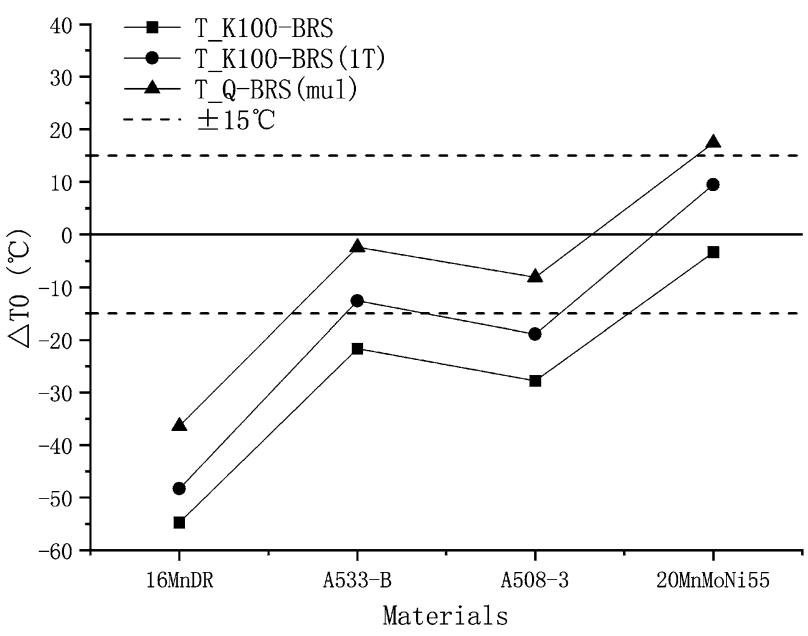

Fig. 10 Deviations of reference temperatures estimated by BR

After determining the range of data selection, an appropriate number of $K_{I C(1 T)}$ data on the temperature axis will be equidistantly selected and the corresponding reference temperature will be calculated by solving Eq. (30). Their results are recorded as $T_{Q-X(m u)}$.

$$
\begin{aligned}
0= & \sum_{i=1}^{i=n} \frac{\delta_{i} \cdot \exp \left\{0.019\left(T_{i}-T_{0}\right)\right\}}{\left[31-K_{\min }+77 \exp \left\{0.019\left(T_{i}-T_{0}\right)\right\}\right]} \\
& -\sum_{i=1}^{i=n} \frac{\left(K_{J d}-K_{\min }\right)^{4} \cdot \exp \left\{0.019\left(T_{i}-T_{0}\right)\right\}}{\left[31-K_{\min }+77 \exp \left\{0.019\left(T_{i}-T_{0}\right)\right\}\right]^{5}}
\end{aligned}
$$

where $K_{\min }$ is $20 \mathrm{MPa} \sqrt{\mathrm{m}}$. Kronecker delta $\delta_{i}$ is 1 for valid data and 0 for invalid data.

The validity of the calculated $T_{Q-X(m u l)}$ are judged according to ASTM E1921.
After completing all the calculation, the deviations of the estimated values are compared. As shown in Figs. 7, 8, 9 and 10, for empirical formulas except BR, all the $T_{Q-X}$ values float around the measured values. Except Euro $20 \mathrm{MnMoNi55}$, the errors within $\pm 10^{\circ} \mathrm{C}$ can be maintained for any other materials. As for $20 \mathrm{MnMoNi55}$, it seems that all estimates of its reference temperature tend to be conservative. Although $T_{Q-X}$ behaves a little conservatively for $20 \mathrm{MnMoNi} 55, T_{Q-X}$ still outperform other predictions. By comparing $T_{K 100-X}$ with $T_{K 100-X(1 T)}$, it is noticed that the calculated $T_{K 100-X}$ still keeps a certain degree of conservatism. Omitting thickness normalization reduces the fluctuation range of the estimated values by almost one third, making the deviation more stable, and greatly simplifying the calculation process. So $T_{K 100-X}$ is more suitable for use. $T_{Q-X(m u l)}$ is calculated by the fracture toughness of $1 \mathrm{~T}$ equivalent thickness and the multi-temperature method. Compared with $T_{K 100-X(17)}$, the results of $T_{Q-X(\mathrm{mul})}$ are always slightly higher by nearly $5{ }^{\circ} \mathrm{C}$. The involvement of multi-temperature method slightly enhances the conservativeness of empirical formulas. However, empirical formulas are conservative enough. This enhancement makes the results of $T_{Q-X(m u l)}$ overly conservative and may affect the correct evaluation of structural integrity. In addition, $T_{Q-X(m u l)}$ uses the multi-temperature method combined with empirical formulas and the calculation process is relatively complex. Considering practicality, $T_{Q-X(m u l)}$ is not priority for the estimation of reference temperature.

The empirical formulas are compared with each other in this paper. As can be seen in Fig. 10, the estimated results of the two-step empirical formula tend to be on the low side and the estimations of $16 \mathrm{MnDR}$ deviate greatly from the tested value. The two-step empirical formula is the first one that should be excluded from selection due to the unstable deviations. Then comparing the one-step empirical formulas in Fig. 7, 8 and 9, it can be seen that these three one-step empirical formulas show similar trends. Obviously, RNB empirical formula is more stable since the deviations are about one quarter smaller than other empirical formulas. Based on Fig. 7 and the conclusion drawn in the previous paragraph, it can be concluded that the estimations of $T_{Q-R N B}$ and $T_{K 100-R N B}$ are the most reliable. The former can provide relatively accurate estimation and the latter can be applied to practical projects with proper conservatism. Both of them are preferred for estimating reference temperature. 


\subsection{Estimations of reference temperature by IGC-parameter}

$\sigma_{f}$ must be known for the calculation of $P_{I G C 1}$. According to the Ref. [26], $\sigma_{f}$ of A533B and 20MnMoNi55 can be defined as $2148 \mathrm{MPa}$ and $2199 \mathrm{MPa}$. For A508-3, due to the lack of sufficient fracture toughness data near $-150^{\circ} \mathrm{C}[26], \sigma_{f}$ is not available and $P_{I G C I}$ is also not considered in this paper. The minimum test temperature of $16 \mathrm{MnDR}$ also does not reach $-150^{\circ} \mathrm{C}$ [24]. The lowest test temperature is $-100^{\circ} \mathrm{C}$. Calculation shows it is impossible to obtain a stable and reliable $\sigma_{f}$ by using these data because of the widely distribution of fracture toughness data at this temperature. So similarly, the $P_{I G C 1}$ of $16 \mathrm{MnDR}$ is also not considered in the following comparisons.

$P_{I G C 1}$ and $P_{I G C 2}$ can be calculated by the Charpy impact energy curves and yield strength formulas of the four materials. The parameters $P_{I G C 1}$ and $P_{I G C 2}$ are used in Eqs. (16-17). The calculated reference temperatures are termed as $T_{Q-I G C 1}$ and $T_{Q-I G C 2}$ respectively and the subscripts indicate that the estimated values are calculated by using IGC parameters. According to the Ref. [19], $T_{Q-28}$, $T_{Q-41}, T_{Q-I G C 1}$ and $T_{Q-I G C 2}$ have a tendency to compensate each other. The mean value of the four data can be closer to the tested value. The mean value is recorded as $T_{Q-M 4}$. If one of the four value is not available, the mean value can also be recorded as $T_{Q-M 3}$. For convenience, $T_{Q-M 3}$ and $T_{Q-M 4}$ are denoted by $T_{Q-M 3 / M 4} \cdot T_{Q-M 3 / M 4}$ will be used as the estimation of reference temperature and compared with experimental value.

This procedure using the mean value as reference temperature is named as the Mean-4 Procedure [19]. To make a significant comparison between $T_{Q-28}, T_{Q-41}, T_{Q-I G C 1}, T_{Q-I G C 2}$ and their mean value $T_{Q-M 3 / M 4}$, these estimations are plotted in the same graph, as shown in Fig. 11.

$T_{41}$ is easily obtained and $T_{Q-I G C 2}$ is available for most materials. Comparing against other estimated values, the $T_{\text {Q-IGC2 }}$ values show high degree of accuracy for the first three materials. However, $T_{\text {Q-IGC2 }}$ behaves a little conservatively for $20 \mathrm{MnMoNi} 55$ and shares similar trend with the estimated results of empirical formulas. As for $T_{Q-I G C 1}$, due to the lack of sufficient data, only the $T_{Q-I G C 1}$ values of two materials can be calculated. Therefore, the existing data cannot show the trend of deviation, and cannot prove that $T_{Q-I G C 1}$ has enough reliability. For $T_{Q-M 3 / M 4}$, it is obvious that the deviations are less than $5{ }^{\circ} \mathrm{C}$ for the first three materials. The deviation of $20 \mathrm{MnMoNi} 55$ is larger than that of other materials. However, the degree of conservatism was decreased and the deviation is reduced to about $8^{\circ} \mathrm{C}$. Generally speaking, the $T_{\mathrm{Q}-M 3 / M 4}$ values show great agreement with the tested $T_{0}$. In terms of the universality of application, $T_{Q-M 3 / M 4}$ can be obtained for all the four materials and the lack or excessive deviation of one component has

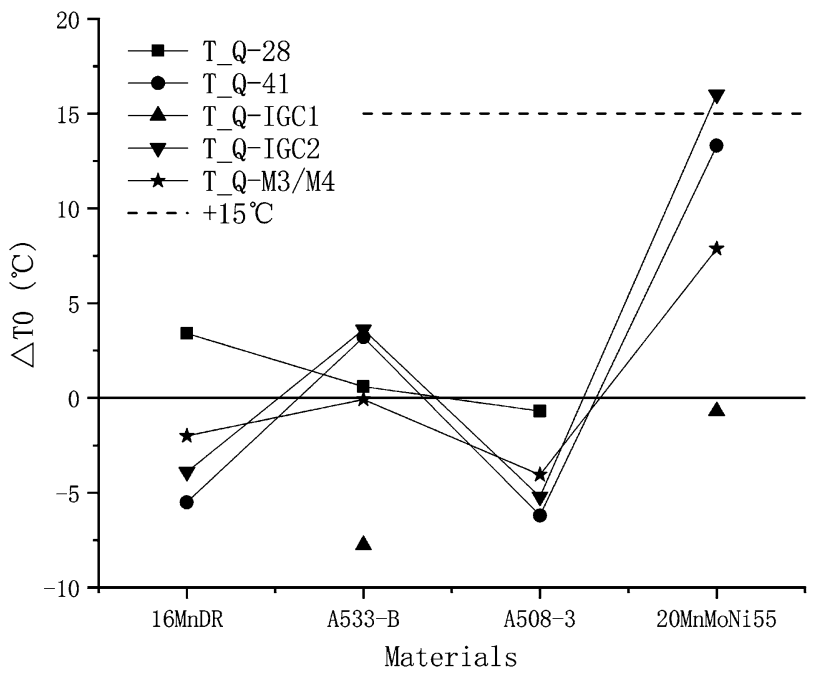

Fig. 11 Deviations of reference temperatures estimated by IGCparameter

limited influence on the mean value. In conclusion, compared with other estimations of IGC parameters, the mean value, $T_{Q-M 3 / M 4}$, was recommended to be a preferred choice for predicting accurate reference temperature.

\section{Conclusions}

In this paper, several methods for estimating reference temperature are researched. Data of four commonly used RPV ferritic steels are applied to estimate the reference temperatures. The reliability of these methods is tested by comparing the test values with the estimated values, so as to find the most suitable estimation methods for practical engineering. The conclusions are as follows:

1. $T_{28}$ has a more stable relationship with reference temperatures than $T_{41}$, and also can be used to obtain more reliable predictions. $T_{Q-28}$ and $T_{0(2 a)}$ calculated by $T_{28}$, can be used as the preferential choice. The former is relatively accurate and the latter is suitable for conservative practical engineering projects. Despite the large deviations, conservative $T_{0(4 a)}$ and accurate $T_{Q-41}$ calculated by $T_{41}$, can also be used as backup options for materials without $T_{28}$.

2. Generally speaking, one-step empirical formulas are more accurate and reliable than two-step empirical formula. For one-step empirical formulas, RNB empirical formula is preferred because of the smaller and more stable deviation. Empirical formulas are sufficiently conservative. However, thickness normalization and combination with the multi-temperature method will improve the conservativeness of empirical 
formulas. Both of them are not preferred because of excessive conservatism. $T_{Q-R N B}$ calculated by empirical formula and non-linear fitting formula gives accurate estimations and $T_{K 100-R N B}$ calculated directly by empirical formula can maintain appropriate conservatism. Both of them are preferential choices for ferritic steel.

3. $T_{Q-M 3 / M 4}$ the mean value of $T_{Q-28}, T_{Q-41}, T_{Q-1 G C 1}, T_{Q-1 G C 2}$ has a wide range of applications and relatively accurate accuracy. The process of calculating mean values weakens the degree of deviation and the requirement for materials. This feature turns $T_{Q-M 3 / M 4}$ into another reliable choice to obtain accurate reference temperature.

Acknowledgements This work was supported by the Fundamental Research Funds for the Central Universities of China (Grant No. PA2019GDPK0054).

\section{Compliance with ethical standards}

Conflict of interest On behalf of all authors, the corresponding author states that there is no conflict of interest.

\section{References}

1. Panin SV, Maruschak PO, Vlasov IV, Sergeev VP, Ovechkin BB, Neifeld VV (2016) Impact toughness of $12 \mathrm{Cr} 1 \mathrm{MoV}$ steel. Part 2 - Influence of high intensity ion beam irradiation on energy and deformation parameters and mechanisms of fracture. Theor Appl Fract Mech. 83:82-92

2. E1921-14a A (2015) Standard test method for determination of reference temperature, $\mathrm{T} 0$, for ferritic steels in the transition range. West Conshohocken, PA 19428-2959

3. Zhu X-K, Joyce JA (2012) Review of fracture toughness (G, K, J, CTOD, CTOA) testing and standardization. Eng Fract Mech 85:1-46

4. Balart MJ, Knott JF (2006) Effects of geometry and flow properties on the fracture toughness of a C-Mn reactor pressure vessel steel in the lower shelf region. Int J Press Vessels Pip 83:205-215

5. Tiwari A, Avinash G, Sunil S, Singh RN, Ståhle P, Chattopadhyay $J$ et al (2015) Determination of reference transition temperature of In-RAFMS in ductile brittle transition regime using numerically corrected Master Curve approach. Eng Fract Mech 142:79-92

6. Ha JS, Fleury E (1998) Small punch tests to estimate the mechanical properties of steels for steam power plant: II. Fracture toughness. Int J Press Vessels Pip 75:707-713

7. Bruchhausen M, Holmström S, Simonovski I, Austin T, Lapetite JM, Ripplinger S et al (2016) Recent developments in small punch testing: tensile properties and DBTT. Theor Appl Fract Mech 86:2-10

8. Scibetta M, Lucon E, van Walle E (2002) Optimum use of broken Charpy specimens from surveillance programs for the application of the master curve approach. Int J Fract 116:231-244

9. Xinping Z, Yaowu S (1996) How to obtain a complete dependence curve of impact toughness or fracture toughness vs temperature on nuclear pressure vessel steels by using only one Charpy-size specimen. Int J Press Vessels Pip 65:187-192

10. Chaouadi R, Puzzolante JL (2008) Loading rate effect on ductile crack resistance of steels using precracked Charpy specimens. Int J Press Vessels Pip 85:752-761

11. Sattari-Far I, Wallin K (2005) Application of Master Curve methodology for structural integrity assessments of nuclear components. SKI (Swedish Nuclear Power Inspectorate) report 2005, p 55
12. Barsom JM, Rolfe ST (1970) Correlations between Klc and Charpy $\mathrm{V}$-notch test results in the transition-temperature range. ASTM STP 466:281-302

13. Rolfe ST, Novak SR (1970) Slow-bend KIC testing of mediumstrength high-toughness steels. ASTM STP 463(463):124-159

14. Pineau A (2008) Modeling ductile to brittle fracture transition in steels-Micromechanical and physical challenges. Int J Fract 150:129-156

15. Rossoll A, Berdin C, Prioul C (2002) Determination of the fracture toughness of a low alloy steel by the instrumented Charpy impact test. Int J Fract 115:205-226

16. Kotousov A, Berto F, Lazzarin P, Pegorin F (2012) Three dimensional finite element mixed fracture mode under anti-plane loading of a crack. Theor Appl Fract Mech 62:26-33

17. Pan J, Chen Z, Hong Z (2019) A novel method to estimate the fracture toughness of pressure vessel ferritic steels in the ductile to brittle transition region using finite element analysis and Master Curve method. Int J Press Vessels Pip 176:103949

18. Wallin K (1989) A simple theoretical Charpy V-KIC correlation for irradiation embrittlement. In: Marriott DL et al (eds) Innovative approaches to irradiation damage and fracture analysis (ASME Pres. Ves. \& Piping Conference, Honolulu, Hawai, (July 1989)), PVPvol. 170. ASME, New York, pp 93-100

19. Sreenivasan PR (2008) Estimation of ASTM E-1921 reference temperature from Charpy tests: Charpy energy-fracture toughness correlation method. Eng Fract Mech 75:5229-5245

20. Sailors R, Corten H (1972) Relationship between material fracture toughness using fracture mechanics and transition temperature tests. ASTM STP 514

21. Roberts R, Newton C (2001) Interpretive report on small-scale test correlations with KIC data. WRC (Welding Research Council) Bulletin No 265

22. Miyata T, Tagawa T (2002) Mezzo-scopic analysis of fracture toughness in steels. Mater Res 5(2):85-93

23. Yu M, Luo Z, Chao YJ (2014) An assessment of mechanical properties of A508-3 steel used in Chinese nuclear reactor vessels. V06ATA020

24. Chen Z, Pan J, Jin T, Hong Z, Wu Y (2018) Estimation of fracture toughness of $16 \mathrm{MnDR}$ steel using Master Curve method and Charpy V-notch impact energy. Theoret Appl Fract Mech 96:443-451

25. GB/T2975-1998 (1998) Steel and steel products-location and preparation of test pieces for mechanical testing. The Standardization Administration of China

26. Yu M, Luo Z, Chao YJ (2015) Correlations between Charpy V-notch impact energy and fracture toughness of nuclear reactor pressure vessel (RPV) steels. Eng Fract Mech 147:187-202

27. Oldfield W (1975) Curve fitting impact test data: a statistical procedure. ASTM Stand News 24-29

28. GB/T229-2007 (2007) Metallic materials-Charpy pendulum impact test method. The Standardization Administration of China

29. Sreenivasan PR, Moitra A, Ray SK, Mannan SL (2004) Predicting reference temperature from instrumented Charpy V-notch impact tests using modified Schindler procedure for computing dynamic fracture toughness. Int J Fract 125:387-403

30. Meshii T, Yamaguchi T (2016) Applicability of the modified RitchieKnott-Rice failure criterion to transfer fracture toughness Jc of reactor pressure vessel steel using specimens of different thicknessesPossibility of deterministic approach to transfer the minimum Jc for specified specimen thicknesses. Theor Appl Fract Mech 85:328-344

31. Sreenivasan PR (2011) Inverse of Wallin's relation for the effect of strain rate on the ASTM E-1921 reference temperature and its application to reference temperature estimation from Charpy tests. Nucl Eng Des 241:67-81

Publisher's Note Springer Nature remains neutral with regard to jurisdictional claims in published maps and institutional affiliations. 